\title{
Boron nitride: A new photonic material
}

M. Chubarov, Henrik Pedersen, Hans Högberg, Stanislav Filippov, J.A. A. Engelbrecht, J. O'Connel and Anne Henry

\section{Linköping University Post Print}

\section{Tweet}

N.B.: When citing this work, cite the original article.

Original Publication:

M. Chubarov, Henrik Pedersen, Hans Högberg, Stanislav Filippov, J.A. A. Engelbrecht, J. O'Connel and Anne Henry, Boron nitride: A new photonic material, 2014, Physica. B, Condensed matter, (439), 29-34.

http://dx.doi.org/10.1016/j.physb.2013.10.068

Copyright: Elsevier http://www.elsevier.com/

Postprint available at: Linköping University Electronic Press http://urn.kb.se/resolve?urn=urn:nbn:se:liu:diva-105563 


\title{
Boron nitride: a new photonic material.
}

\author{
M. Chubarov ${ }^{1, \mathrm{a}}$, H. Pedersen ${ }^{1, \mathrm{~b}}$, H. Högberg ${ }^{1, \mathrm{c}}$, S. Filippov ${ }^{1, \mathrm{~d}}$, J.A.A. Engelbrecht ${ }^{2, \mathrm{e}}$, \\ J. O'Connel ${ }^{2, \mathrm{f}}$ and $\underline{\mathrm{A} . \text { Henry }}{ }^{1, \mathrm{~g}}$
}

${ }^{1}$ Dep. of Physics, Chemistry and Biology, Linköping University, SE-581 83 Linköping, Sweden

${ }^{2}$ Nelson Mandela Metropolitan University, Port Elizabeth, South Africa

Corresponding author e-mail address: g) anne.henry@liu.se, Tel: +4613282414, Fax: +4613142337

a)mihcu@ifm.liu.se, b) henke@ifm.liu.se,c)hanho@ifm.liu.se,d) stafi@ifm.liu.se,

e) Japie.Engelbrecht@nmmu.ac.za and f) jacques.oconnell@gmail.com

\section{Abstract}

Rhombohedral Boron Nitride (r-BN) layers were grown on sapphire substrate in a hot-wall chemical vapor deposition reactor. Characterization of these layers is reported in details. Xray diffraction (XRD) is used as a routine characterization tool to investigate the crystalline quality of the films and the identification of the phases is revealed using detailed pole figure measurements. Transmission electron microscopy reveals stacking of more than 40 atomic layers. Results from Fourier Transform InfraRed (FTIR) spectroscopy measurements are compared with XRD data showing that FTIR is not phase sensitive when various phases of $\mathrm{sp}^{2}$-BN are investigated. XRD measurements show a significant improvement of the crystalline quality when adding silicon to the gas mixture during the growth; this is further confirmed by cathodoluminescence which shows a decrease of the defects related luminescence intensity.

\section{Keywords}

Boron Nitride, epitaxy, XRD, TEM, FTIR, Cathodoluminescence

\section{Introduction}

Boron nitride (BN) is today the least investigated and hence understood III-nitride material and now gathers researchers' interest due to its amazing properties and close similarities with the polytypes of carbon. $\mathrm{BN}$ is a wide band gap semiconductor which can be used for 
optoelectronic devices working in the UV range; the band gap of $\mathrm{BN}$ is comparable to $\mathrm{AlN}$, but its properties are not fully understand since various values have been reported for the band gap energies, in both experimental and theoretical studies, with discussion regarding its nature (direct or indirect). Ultra-violet emission at $215 \mathrm{~nm}(5.765 \mathrm{eV})$ has been observed from hexagonal $\mathrm{BN}$ (h-BN) small single crystals obtained under high- pressure and temperature conditions [1]. Recently, h-BN epilayers grown by metal organic chemical vapor deposition (MOCVD) were demonstrated to exhibit a very efficient band gap emission close to $5.5 \mathrm{eV}$, which was found polarized perpendicular to the c-axis and with intensity two orders of magnitude higher than AlN. The estimated band edge absorption coefficient was also higher than that of AlN [2]. Kobayashi et al. reported the growth of a $500 \mathrm{~nm}$ thick h-BN film on Ni (111) substrate, which exhibits a near-band-gap ultraviolet luminescence at $227 \mathrm{~nm}$ [3]. This group proposed that h-BN film can be used as a release layer for mechanical transfer of AlGaN-based devices [4] due to its ability of easy exfoliation. These various results are good indicators for $\mathrm{BN}$ to be a candidate for optical applications, especially in the deep UV region.

The synthesis of semiconducting BN epilayers of high crystalline quality and with controlled electrical conductivity is urgently needed for the fundamental understanding of this interesting material towards the exploration of possible applications. However, high quality epitaxial thin films of $\mathrm{BN}$ are difficult to grow, and in the literature majority of reports shows that the grown films contain significant amounts of various phases. It should be noted that BN can form compounds with either $\mathrm{sp}^{3}$-hybridized (cubic (c-BN) or wurtzite (w-BN) form) or $\mathrm{sp}^{2}$-hybridized (h-BN or rhombohedral (r-BN) phase) bonds. For the most investigated phase of $\mathrm{BN}$, viz. c-BN, generally polycrystalline films are reported. Ion bombardment during the deposition process is regarded to be necessary for the nucleation of c-BN films and maintaining the $\mathrm{sp}^{3}$-hybridization of the bonds $[5,6]$. Hence, energetic ion-assisted physical vapor deposition including $\mathrm{rf}$ and magnetron sputtering as well as ion-beam assisted deposition have been the choice for the c-BN deposition. This means that, due to the growth mechanism, the obtained BN material contains many structural defects, fact which results in poor crystalline quality.

The chemical route represents a more gentle deposition process and CVD techniques operated at high energy regime like plasma enhanced CVD techniques have been used. Very few attempts have been reported on the BN synthesis by processes without the use of energetic deposition and those approaches seem to favor the formation of $\mathrm{sp}^{2}$ bonded phases.

We recently reported the epitaxial growth of $\mathrm{sp}^{2}-\mathrm{BN}$ films on sapphire with an AlN buffer layer using thermally activated CVD [7] and using X-ray diffraction (XRD) pole figure 
measurements, we demonstrated that the BN films had a twinned rhombohedral crystal structure [8]. High quality $\mathrm{r}-\mathrm{BN}$ is also found to be promoted by using a $\mathrm{Si}$ containing environment during growth [9].

In this paper we describe in more detail results obtained from the characterization of this material and especially reveal some of the difficulties encountered.

\section{Material and methods}

Epitaxial growth of r-BN is done in a horizontal hot-wall chemical vapor deposition reactor using ammonia $\left(\mathrm{NH}_{3}\right)$ and triethyl boron (TEB) as nitrogen and boron precursors, respectively, and (0001) sapphire as substrate. An AlN buffer layer is formed by in-situ nitridation prior to the $\mathrm{BN}$ growth $[7,8]$. The growth is carried out at low pressure (typically 100 mbar or lower) with $\mathrm{H}_{2}$ as carrier gas in the temperature range of $1200-1500{ }^{\circ} \mathrm{C}$ and with an N/B-ratio close to 700.

XRD was applied for the characterization of the deposited BN layers and first routinely in the Bragg-Brentano geometry using the $\mathrm{Cu} K \alpha_{1}$ radiation. This was done first in a powder diffractometer (PANalytical X'Pert PRO) for low-resolution $\theta-2 \theta$ measurements, but a PANalytical EMPYREAN MRD instrument was used for high resolution $\theta-2 \theta$ and $\omega$ measurements as well as to record pole figures. Cross-sectional specimens for transmission electron microscopy (TEM) were produced by two different approaches; either by conventional mechanical polishing followed by $\mathrm{Ar}^{+}$ion milling at $5 \mathrm{keV}$ at a grazing angle of $5^{\circ}$ or by milling and thinning the sample using a FEI Helios NanoLab 650.

For phase identification we also applied Fourier Transform InfraRed (FTIR) spectroscopy, which is a non-destructive and rapid technique and can be performed either in reflection or transmission mode. We measured FTIR reflectance in a Brucker $80 \mathrm{~V}$ FTIR/Raman spectrometer with an incidence angle of $15^{\circ}$ and $4 \mathrm{~cm}^{-1}$ resolution. Micro-Raman measurements were performed in LabRAM HR800 equipped with a single grating monochromator with an excitation at $659 \mathrm{~nm}$ of approx $60 \mathrm{~mW}$ and 100x lens. Cathodoluminescence (CL) measurements were performed in a Leo 1550 field-emission gun scanning electron microscope equipped with a MonoCL2 system (Oxford Res. Instr.) at low temperature (typically $\mathrm{T}<10 \mathrm{~K}$ ) and using electron beam energy of $10 \mathrm{keV}$.

\section{Results and discussion}

\subsection{Growth results and XRD}


At optimal growth conditions $\left(\mathrm{T}=1500^{\circ} \mathrm{C}, 70 \mathrm{mbar}, \mathrm{N} / \mathrm{B}=650\right)$ rhombohedral epitaxial layers were obtained as proven by XRD analysis and particularly Pole figure measurements revealed a twinned structure of the rhombohedral BN (r-BN) films. Fig.1.a illustrates a typical XRD $\theta-2 \theta$ scan diffractogram of one of the layers. The sharp peak of highest intensity at $41.69^{\circ}$ is associated to the reflection from the (0006) plane of the sapphire substrate and more weakly the reflection from the $\mathrm{Al}_{2} \mathrm{O}_{3}$ (0003) plane is detected at $20.50^{\circ}$. Note that this peak corresponds to a forbidden reflection which, as the name implies and according to the structure factor of the sapphire lattice, should not be visible in diffraction experiments but becomes visible due to the kinetic scattering of the X-rays. Contribution from the AlN buffer is also observed at $36.04^{\circ}$ corresponding to the (0002) plane reflection; the $0002 \mathrm{AlN}$ peak is rather broad which suggests that the buffer layer is thin and strained. We have demonstrated that high quality relaxed AlN layer which exhibits very sharp 0002 peak in $\theta-2 \theta$ scan XRD does not promote the growth of r-BN and only turbostratic $\mathrm{BN}(\mathrm{t}-\mathrm{BN})$ is deposited [7]. $\mathrm{t}-\mathrm{BN}$ differs from the two crystalline $\mathrm{sp}^{2}$ phases of $\mathrm{BN}, \mathrm{h}-\mathrm{BN}$ and $\mathrm{r}-\mathrm{BN}$, by a larger spacing between the basal planes $(\geq 3.35 \AA$ ) and a lack of stacking sequence. Peaks observed in the range from $44^{\circ}$ to $46^{\circ}$ originate from the sample holder and the sharp peak at $37.51^{\circ}$ is due to the $\mathrm{CuK}_{\beta}$ line diffraction on the sapphire (0006) planes. The peaks associated with r-BN are at the $2 \theta$ angles of $26.71^{\circ}$ and $55.06^{\circ}$ (with an error of $+/-0.01^{\circ}$ ) which correspond to reflections from the (0003) and (0006) planes, respectively. It should noticed here that reflections from the (0002) and (0004) planes of h-BN will also give rise to XRD peaks at the same positions since the two $\mathrm{sp}^{2}-\mathrm{BN}$ phases, $\mathrm{r}-\mathrm{BN}$ and $\mathrm{h}-\mathrm{BN}$, have similar spacing between the basal planes (3.325 $\AA$ and $3.33 \AA$ for h-BN and r-BN, respectively) but differ with their stacking sequences ( $\mathrm{ABAB} \ldots$ and $\mathrm{ABCABC} \ldots$ stacking for $\mathrm{h}-\mathrm{BN}$ and $\mathrm{r}-\mathrm{BN}$, respectively). To distinguish between these two $\mathrm{sp}^{2}$ phases a first approach is possible with the intensity ratio of these two peaks: the peak at lower angle $\left(26.71^{\circ}\right)$ is 25 times higher in intensity than the peak at higher angle for the r-BN phase and about 17 times for h-BN bulk material [10]. For our layers when a sharp and intense peak is observed at or very close to $26.71^{\circ}$, the peak at $55.06^{\circ}$ can be distinguished from the background and the intensity ration of the two peaks is typically 25 , and even as high as 27 for few layers. A second technique is the use of XRD pole figure measurements which reveal that the r-BN layers have a twinned rhombohedral structure $30^{\circ}$ rotated with respect to the sapphire substrate [8]. The rotation is in fact due to the $30^{\circ}$ rotation of the AlN buffer layer on sapphire [11]; the $\mathrm{sp}^{2}-\mathrm{BN}$ crystal grows directly on the AlN template. During the pole figure measurements an angle $2 \theta$ of $50.15^{\circ}$ and $45.57^{\circ}$ for $\mathrm{h}-\mathrm{BN}$ and 
r-BN, respectively, corresponding to the reflection of the $\{01 \underline{12}\}$ planes has been used. For the case of h-BN six peaks around the $\Phi$ axis should be observed with a separation of $60^{\circ}$, (Fig.2.a) whereas only 3 peaks with $120^{\circ}$ separation are predicted for r-BN (Fig.2.c). In the measurement using a $2 \theta$ angle of $50.15^{\circ}$ (Fig.2.b) only three distinct peaks are observed. Due to the limited thickness and low electron density of the $\mathrm{sp}^{2}-\mathrm{BN}$ film and the fact that the measurement is done without monochromatic beam, contribution for the substrate can be expected which will complicate the interpretation of the results. This is the case here since sapphire has a diffraction from $\{02 \underline{2} 4\}$ at $52.533^{\circ}$ and those three peaks are attributed to the sapphire substrate. With a $2 \theta$ angle of $45.57^{\circ}$ (Fig.2.d) 6 weak peaks are visible in the pole figure instead of 3 predicted for $\mathrm{r}-\mathrm{BN}$. This behavior reveals a twinning of the rhombohedral crystal structure.

More close investigation of the 0003 peak (Fig.3), reveals that the peak is a contribution of two different peaks: a sharp peak at $26.71^{\circ}$ with a full width at half maximum (FWHM) of only $0.16^{\circ}$, being the smallest reported value for any $\mathrm{sp}^{2} \mathrm{BN}$ phase, to the best of our knowledge, and a weak and broad $\left(\mathrm{FWHM}=1^{\circ}\right)$ peak at $26.3^{\circ}$ which is associated to the less ordered form of $\mathrm{sp}^{2}-\mathrm{BN}$, the turbostratic $(\mathrm{t}-\mathrm{BN})$. The $\mathrm{t}-\mathrm{BN}$ peak at $26.3^{\circ}$ appears when the growth is done at unfavorable conditions or when the r-BN lattice is damaged due to e.g. stress. An example of XRD pattern where only t-BN is visualized is given in Fig.1.b.

Since the 0003 peak of bulk r-BN is expected to be at $26.717^{\circ}$ [10] XRD measurements show that we did obtain high quality r-BN films. However, this is achieved only with low growth rate (less than $200 \mathrm{~nm} / \mathrm{h}$ ). Increasing the $\mathrm{B} / \mathrm{H}_{2}$ ratio in the gas phase allows an increase of the growth rate, but with a degradation of the crystal quality as observed by an increasing basal plane spacing; the 0003 peak of $\mathrm{r}-\mathrm{BN}$ is shifted from $26.72^{\circ}$ to $26.56^{\circ}$ for the samples grown at $\mathrm{B} / \mathrm{H}_{2}$ ratios of $0.01 \%$ and $0.07 \%$, respectively. This increase of the basal plane spacing is proposed to be favored by incorporation of impurities such as carbon and/or oxygen atoms. Moreover XRD measurement reveals that films grown in presence of Si atoms exhibit better crystal quality, typically as presented in Fig.1.a, than those grown without $\mathrm{Si}$ which show generally a turbostratic structure as shown in Fig.1.b. When trying different types of coating ( $\mathrm{SiC}, \mathrm{TaC}$ and hot-pressed $\mathrm{BN}$ powder) for the susceptor we found that any presence of water and/or oxygen during BN CVD leads to deposition of mainly boron oxide films via incorporation of species containing a very strong B-O bond. We showed by thermodynamic calculations that these B-O bonds are formed already in the gas phase. Any oxygen contamination of the growth chamber thus prohibits the growth of high quality BN [12]. 
XRD $\omega$-scan measurements have also been performed and confirm the formation of r-BN. The peak is rather broad with a FWHM of $1.1^{\circ}$ which reveals stress in the material (see Fig.3.b).

\subsection{SEM, TEM and exfoliation problem}

The surface of an r-BN layer as observed with SEM is shown in Fig.4.a. Similar morphology has been observed for all investigated layers irrespectively of the $\mathrm{sp}^{2}$ phase, $\mathrm{r}-\mathrm{BN}$ or $\mathrm{t}-\mathrm{BN}$. The surface is rather rough with nanosize features on the surface. When manipulating the material an exfoliation is easy observed and examples with cross section SEM are given in Fig.4. In Fig.4.b part of the r-BN epilayer is lifted from the substrate and a rather dense r-BN material is observed with a thickness of about $2 \mu \mathrm{m}$ covered by nanorods which could be as high as $2 \mu \mathrm{m}$ without specific ordering or orientation. This is also visualized in Fig.4.c where part of the layer is still attached to the substrate (left and right part on the image) whereas others parts are exfoliated from the substrate, as at the center of this picture which shows an undulation of the layer. The total thickness of the dense layer is less than $1 \mu \mathrm{m}$ whereas nanosized rods reach few $\mu \mathrm{m}$ high. A second example is given in Fig.4.d, which occurred when preparing the sample for TEM investigation using polishing technique. The $400 \mathrm{~nm}$ thick layer is lifted from the substrate and a total ripple of the layer is observed. This shows the difficulty of preparing samples for further investigations such as TEM. As we have reported [8] in the case of the growth of high quality r-BN some amount of the t-BN can be formed and observed at the interface r-BN/AlN. t-BN could be formed due to the deterioration of the crystal structure which is mainly due to the difference in the in-plane linear thermal expansion coefficient between $\mathrm{sp}^{2}$-BN and AlN/sapphire. While sapphire and AlN have a positive inplane linear thermal expansion coefficient which increases with temperature, $\mathrm{sp}^{2}-\mathrm{BN}$ has a negative coefficient in a wide temperature range. This results in severe stress in the grown material as it cools down after deposition at high temperature. In addition at high temperature (typically more than $1400{ }^{\circ} \mathrm{C}$ ) voids can appear in the sapphire substrate at the interface $\mathrm{AlN} / \mathrm{Al}_{2} \mathrm{O}_{3}$ enhancing the exfoliation [13]. However, we succeeded to prepare specimens for cross sectional TEM and the image (Fig.4.e) shows up to $40 \mathrm{BN}$ layers which is the largest number reported up till now in the literature, to the best of our knowledge. However, some stacking faults and grain boundaries are seen and other defected areas are also observed associated to the $\mathrm{t}-\mathrm{BN}$ structure.

\subsection{Optical characterization}


FTIR measurements have been widely used for the characterization of BN, particularly for the phase composition when mixture of $\mathrm{sp}^{2}$ and $\mathrm{sp}^{3}$ forms appears. The peaks of interest for h-BN are at $\sim 783$ and $828 \mathrm{~cm}^{-1}$ corresponding to the out of plane vibration modes (B-N-B mode, transversal and longitudinal) and $\sim 1367$ and $1610 \mathrm{~cm}^{-1}$ which are from the in-plane stretching vibrations (B-N mode) [14]. Polarization dependence [15] as well as pressure dependence [16] have been reported for the $\mathrm{sp}^{2} \mathrm{~h}-\mathrm{BN}$. However, to the best of our knowledge, r-BN has not been considered. As its crystalline structure is very close to that of h-BN we would expect similarity for the optical properties of these both phases. Typical spectra recorded for few films are shown in Fig. 5 where the main structure below $1000 \mathrm{~cm}^{-1}$ is related to the sapphire substrate. The contribution from the AlN buffer layer is observed close to $675 \mathrm{~cm}^{-1}$, and can be slightly shifted in wavenumber position from sample to sample. This peak is related to the transversal optic mode and the slight shift observed from the bulk value $\left(671.6 \mathrm{~cm}^{-1}\right)$ [16] is interpreted as a consequence of the strain existing in the AlN buffer layer which also is at the origin of the broadening of the 0002 AlN peak observed at $36.04^{\circ}$ in the XRD $\theta-2 \theta$ scan (see Fig.1). In the FTIR spectra the two peaks observed at 802 and $1370 \mathrm{~cm}^{-1}$ as indicated by an arrow in Fig.5 are associated to the out-of-plane bending vibration and in-plane stretching modes in r-BN. In addition, the B-N-B mode sometime has a broad background as in the case of Fig.5.d) which is not understood yet. The splitting of the B-N observed in Fig.5.a and b) is attributed to the two optical modes, transverse and longitudinal.

Most important is the appearance of these peaks which is found to be independent of the $\mathrm{sp}^{2}$ phase ( $\mathrm{r}-\mathrm{BN}$ or $\mathrm{t}-\mathrm{BN}$, in our case) when compared with the XRD analysis. The labeling of each layer is noted in Fig.5 together with the results obtained from the XRD $\theta-2 \theta$ scans: as first example the curve a) and b) in Fig.5 are very similar whereas their associated XRD diffractograms differ in the same way than the both displayed in Fig.1. A second example is for the curve Fig.5.d, where the B-N-B mode is well observed whereas the B-N vibration is not detected and the XRD pattern for this layer is characteristic for r-BN. As mentioned the addition of small amount of $\mathrm{SiH}_{4}$ to the gas mixture during $\mathrm{sp}^{2}$-BN deposition improves the crystalline quality of the growing film and contributes to the growth of high quality epitaxial r-BN instead of less ordered t-BN film. SIMS composition measurements revealed an average concentration of $\mathrm{Si}$ in the low $10^{19} \mathrm{~cm}^{-3}$ range [9]. Nevertheless there are no indications of Si$\mathrm{N}$ bonds in the FTIR spectra where a peak at about $850 \mathrm{~cm}^{-1}$ is expected.

Using Raman spectroscopy the characteristic peak for the in plane vibrations of $\mathrm{sp}^{2}$ hybridized B-N is found at $1369 \mathrm{~cm}^{-1}$ (Fig.6). The peak is almost at the same position than previously reported for h-BN [14], similar peak position is expected for $\mathrm{r}-\mathrm{BN}$ material since 
same chemical bonds exist in h-BN and r-BN. In addition as indicated in Fig.6, the FWHM is found to be $30 \mathrm{~cm}^{-1}$ which reveals the high crystalline quality of the r-BN grown layer.

Low temperature CL measurements show difference in the luminescence spectra for layers grown with or without intentional addition of Si during the growth. Fig.7 shows the CL spectra recorded from two different layers and the inset of the figure shows the same spectra but with normalized intensity. First the total intensity is found to be 20 times higher when there is no $\mathrm{Si}$ addition to the gas mixture during the growth. We should notice here that due to the experimental set-up it was not possible to record the spectra at higher energy; the near band gap emission is thus probably not visualized in the recorded range. Moreover various bands are observed. The band at about $350 \mathrm{~nm}$ which is the most intense for the sample grown without addition of $\mathrm{Si}$ and decreases significantly in intensity when small amount of $\mathrm{Si}$ is added to the gas mixture, is typically assigned to carbon and oxygen related luminescence in h-BN [17]. In h-BN a band at $400 \mathrm{~nm}$ was assigned to native surface defects which are sensitive to oxygen ambient [18]. We observe other broad bands around 430, 505 and $570 \mathrm{~nm}$ which prove that the quality of the material needs further improvement.

\section{Conclusions}

Various characterization techniques, structural and optical, can be used to investigate the quality of $\mathrm{sp}^{2}$-BN layers. Sharp XRD peaks at $26.71^{\circ}$ and $55.06^{\circ}$ are associated to the reflection from the (0003) and (0006) planes of the r-BN phase, whereas larger peak at $26.3^{\circ}$ is related to t-BN form. Pole figure measurements reveal a twinning of the $\mathrm{r}-\mathrm{BN}$ structure. Using FTIR measurements vibration modes are observed at 802 and $1370 \mathrm{~cm}^{-1}$ and with Raman spectroscopy the in-plane vibration is found at $1369 \mathrm{~cm}^{-1}$. However FTIR and XRD conducted on the same layers give different results; their complimentary is needed for a more fundamental understanding of the properties of the material especially for the r-BN phase which has not been investigated until now. Improvement of the crystalline structure is also confirmed by cathodoluminescence measurements which show a decrease of the intensity of the luminescence associated to defects for samples grown with addition of silane to the gas phase. Special preparation as for TEM or cross section experiments can influence the mechanical properties and induces change or destruction of the original phase. More work is needed to understand, more deeply, the growth mechanism of r-BN and its structural, physical and optical properties and thereby push the state-of-the-art quality of r-BN.

\section{Acknowledgement}


The Swedish Research Council (VR 621-2009-5264) and "Carl Tryggers Stiftelse" are gratefully acknowledged for financial support as well as the South Africa/Swedish Research Cooperation Program. Dr. V. Darakchieva and Dr. M. Xie are acknowledged for assistance with the CL measurements.

\section{References}

1. K. Watanabe, T. Taniguchi and H. Kanda, Nature Materials 3, 2004, 404-409

2. X.K. Cao, S. Majety, J. Li, J.Y. Lin and H.X. Jiang, Proc. of SPIE 8631, 2013, 863128-9

3. Y. Kobayashi, T. Akasaka, and T. Makimoto, and N. Matsumoto, J. Cryst. Growth 310, 2008, 5048-5052

4. Y Kobayashi , K. Kumakura, T. Akasaka and T. Makimoto, Nature, 484, 2012, 223-227

5. P.B. Mirkarimi, K.F. McCarty and D.L. Medlin, Mat. Sci. \& Eng. R21, 1997, 47-100

6. W.J. Zhang, Y.M. Chong, I. Bello and S.T. Lee, J. Phys. D, 40, 2007, 6159-6174

7 M. Chubarov, H. Pedersen, H. Högberg, and A. Henry, Cryst. Growth and Des. 12, 2012, $3215-3220$

8. M. Chubarov, H. Pedersen, H. Högberg, V. Darakchieva, J. Jensen, Per O. Å. Persson, and A. Henry, Phys. Status Solidi RRL 5, 2011, 397-399

9. M. Chubarov, H. Pedersen, H. Högberg, and A. Henry, CrystEngComm 15, 2013, 455-458

10. Joint Committee on Powder Diffraction Standards, Swarthmore, PA, pattern 34 - 0421; pattern $45-1171$

11. H. Fukuyama, S. Kusunoki, A. Hakomori, and K. Hiraga, J. Appl. Phys. 100, 2006, $024905,1-7$

12. H Pedersen, M. Chubarov, H. Högberg, J. Jensen, and A. Henry, Thin Solid Films 520, 2012, 5889-5893

13. Y. Kumagai, Y. Enatsu, M. Ishizuki, Y. Kubota, J. Tajima, T. Nagashima, H. Murakami,

K. Takada, and A. Koukitu, J. Cryst. Growth, 312, 2010, 2530-2536

14. R. Geick, C.H. Perry and G. Rupprecht, Phys. Rev. 146, 1966, 543-547

15. M.C. Polo, M. Ben el Mekki, J.L. Andújar, N. Mestres and J. Pascual, Diam. Rel. Mat. 6, $1997,1550-1554$

16. J.A. Sanjurjo, E. López-Cruz, P. Vogl and M. Cardona, Phys. Rev. B 28, 1983, 45794584

17. T. Taniguchi and K. Watanabe, J. Cryst. Growth, 303, 2007, 525-529

18. V. Korsaks, B. Berzina and L. Trinklere, Latv. J. of Phys, and Techn. Sci. 2, 2012, 57- 61 
Figure captions

Fig.1: X-Ray diffractions pattern measured in the Bragg-Brentano configuration for a) a high quality epitaxial r-BN and b) a t-BN layer.

Fig.2: (102) pole figures of a) $\mathrm{h}-\mathrm{BN}$ as predicted for a angle $\theta=51.15^{\circ}$ and b) as measured and (012) pole figures of c) r-BN as predicted for an angle $\theta=45.57^{\circ}$, and d) as measured. The red circles in the measured pole figure are for a better visualization of the peak positions.

Fig.3: a) High resolution XRD $\theta-2 \theta$ scan over the 0003 BN peak showing the contribution of r-BN and t-BN and b) $\omega$-scan for the same layer. The peak positions obtained from the fitting procedure are indicated in the figure for the $\mathrm{r}-\mathrm{BN}$ and $\mathrm{t}-\mathrm{BN}$, respectively. Their FWHM are found to be $0.16^{\circ}$ and $1^{\circ}$, respectively. For the w-scan the FWHM is $1.1^{\circ}$.

Fig.4: a) Top view SEM image of a r-BN layer, b) and c) cross section SEM images showing exfoliation from the substrate, d) TEM image illustrating the total ripple of the r-BN layer and e) cross section TEM of a r-BN layer showing more than 40 basal planes.

Fig.5: FTIR spectra of 4 different BN layers. The spectra have been shifted along the Y-axis for a better visualization. The label of each layer is indicated as well as the phase association resulting form the XRD analysis.

Fig.6: $\mu$-Raman scattering spectrum of r-BN film.

Fig.7: CL spectra of layers a) grown with addition of Si to the gas mixture and b) without addition of $\mathrm{Si}$. The other growth parameters were the same $\left(\mathrm{T}=1500^{\circ} \mathrm{C}, \mathrm{p}=70 \mathrm{mbar}, \mathrm{B} / \mathrm{N}=\right.$ 650 and $\left.\mathrm{B} / \mathrm{H}_{2}=0.02 \%\right)$. 
Fig.1

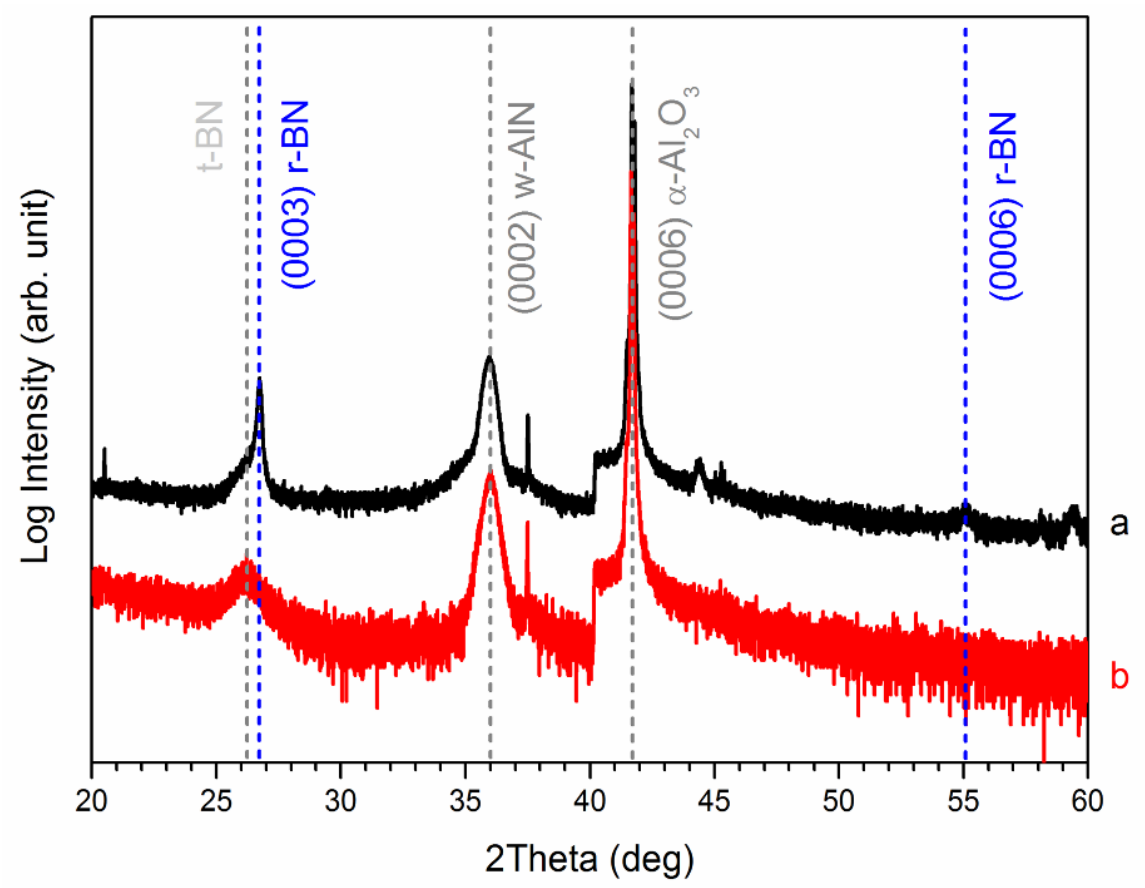


Fig. 2
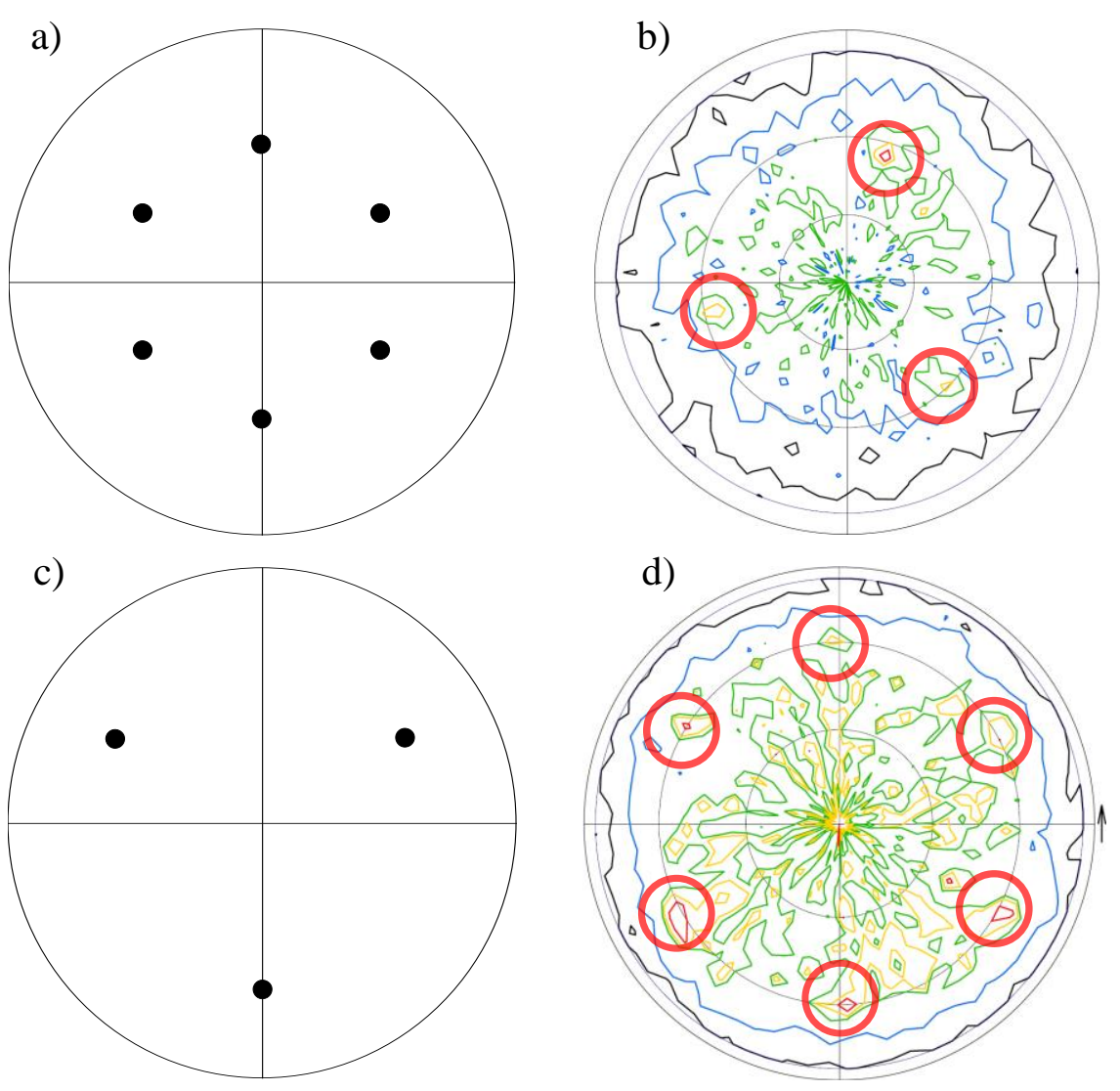
Fig. 3

a)

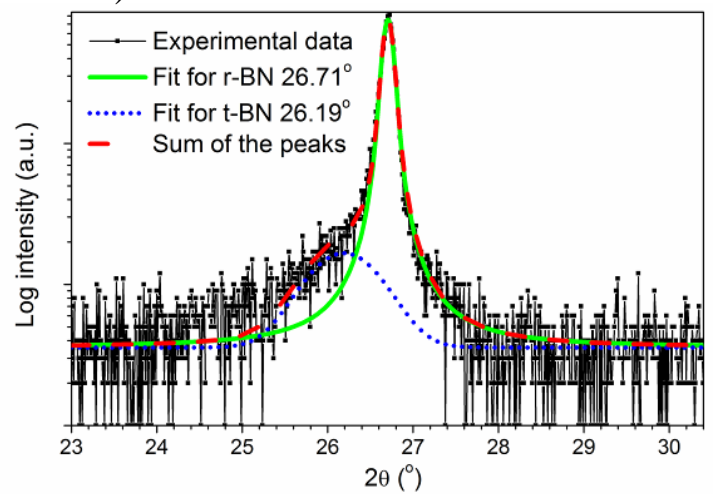

b)

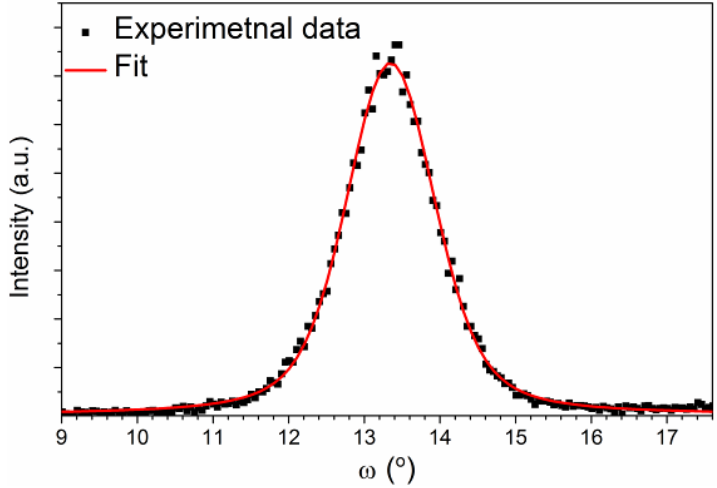


Fig.4:
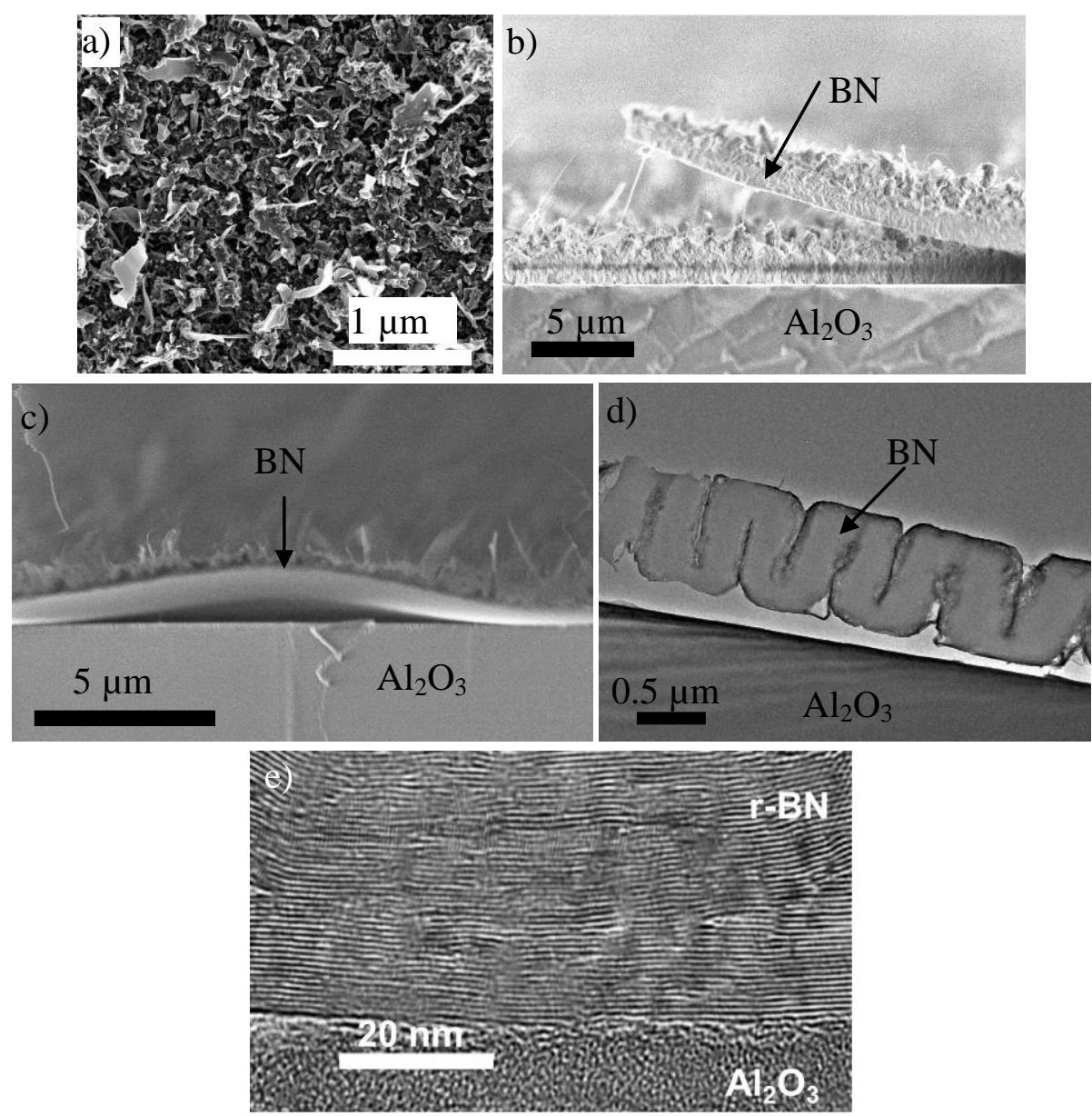
Fig.5

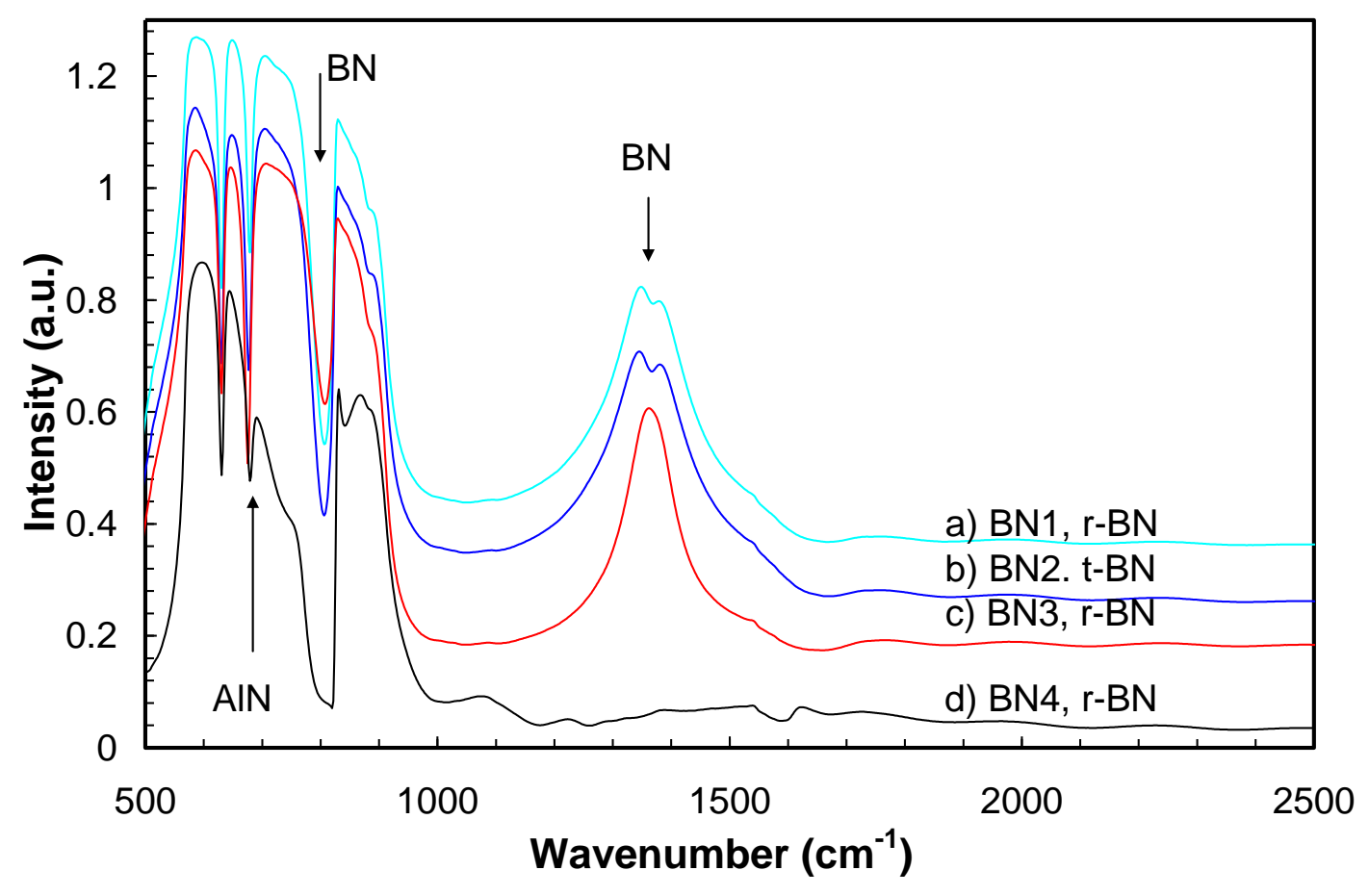


Fig.6

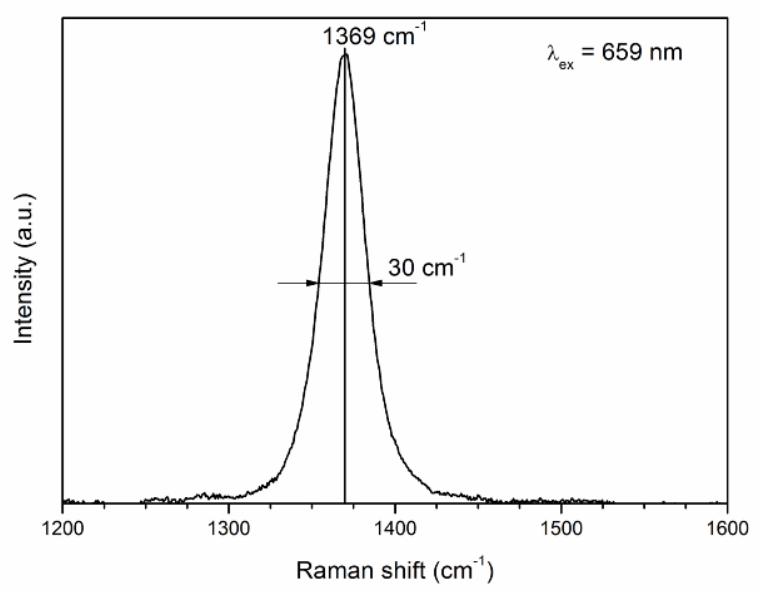


Fig.7

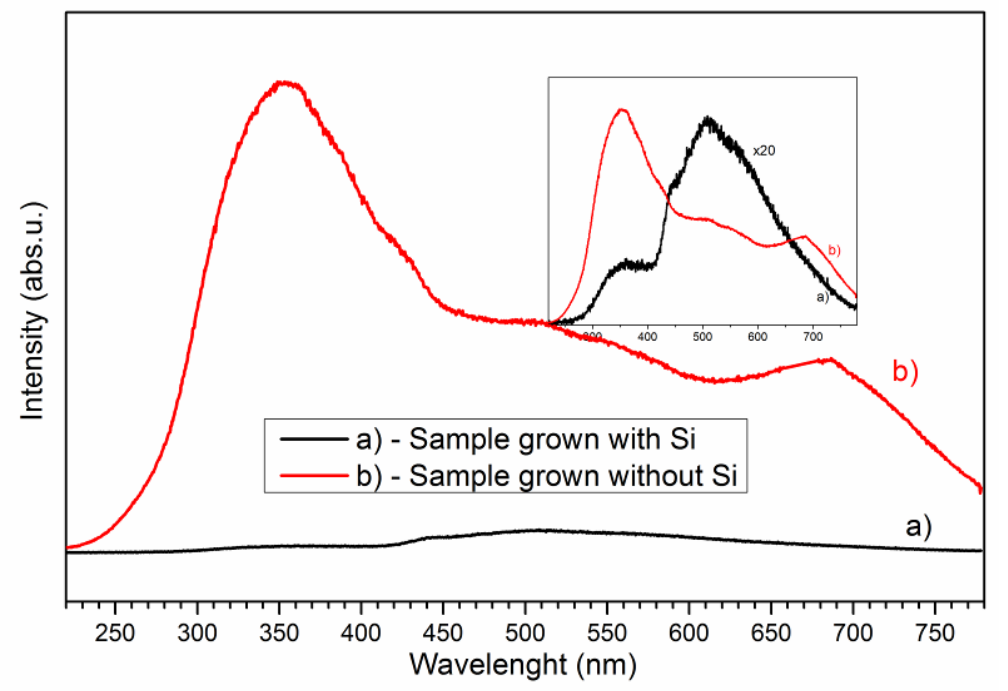

